\title{
Foucault pendulum revisited, the determination of precession angular velocity using Cartesian coordinates
}

\author{
José A. Giacometti ${ }^{*} 1,20$ \\ ${ }^{1}$ Universidade de São Paulo, Instituto de Física de São Carlos, 13566-590, São Carlos, SP, Brasil. \\ ${ }^{2}$ Universidade Estadual Paulista, Faculdade de Ciências e Tecnologia, 19060-900, Presidente Prudente, SP, Brasil.
}

Received on June 14, 2019. Accepted on December 21, 2020.

\begin{abstract}
A review on the Foucault pendulum motion is presented using Cartesian coordinates for the ideal case and for small amplitude of oscillations. The choice of referential frames, the formulation and solution of Newton's differential equation for the non-inertial frame of the Earth and the validity of the approximations used to simplify the determination of the solution are given. Using the angular position of the trajectory cusps, a new method to determine the precession angular velocity of the Foucault pendulum is shown. The pendulum bob trajectories and velocities for the referential frame in rotation with the Earth as well as for the inertial frame are given and the Chevilliet theorem was demonstrated. In addition, the pendulum bob trajectories are shown when an initial velocity is impinged in the direction perpendicular to the pendulum oscillation plane.
\end{abstract}

Keywords: Foucault pendulum, precession velocity.

\section{Introduction}

In 1851 in the Pantheon in Paris, the French physicist Jean Bernard Léon Foucault made the public presentation of his pendulum [1. The experiment was the first demonstration of the Earth's rotation without requiring observation of the apparent motion of stars. Foucault constructed a suspended device that avoided mechanical interference in the pendulum motion, allowing for a careful analysis of its oscillation plane. He made visible the effect of the Coriolis force, that is, due to the Earth's rotation, the oscillation plane of the pendulum changes direction very slowly, in the so-called "precession movement". The Foucault pendulum produced a great impact [2]; the experiment was repeated in many countries (USA and other European countries) and put to an end the philosophical discussions about Earth's motion. In Brazil, also in 1851, C. B. de Oliveira [3] reported the failure to observe the precession of the Foucault pendulum in Rio de Janeiro. Foucault's pendulum continues to stimulate public curiosity. Since 1851 much more than a hundred articles have addressed its use for didactic purposes in classical mechanics, various theoretical aspects on the pendulum motion as well as technical details of its construction. In the poll conducted by Physics World Magazine 4, it was considered one of the ten most beautiful experiments in physics. A historical summary about Earth's rotation is provided in reference [5] and Foucault's biography can be found in references $[5,6]$.

It is noteworthy to recall that Auguste Bravais [7, 8] also in Paris in 1851, carried out another experiment

\footnotetext{
$\overline{\text { * Correspondence address: }}$ giacometti@ifsc.usp.br
}

to demonstrate Earth's rotation. He measured the time difference between the periods when a conical pendulum, 10 meters long, was set to rotate clockwise and counterclockwise. However, despite its successful experiment to also demonstrate Earth's rotation, the Bravais pendulum was almost forgotten by the scientific community [9, 10]. His experiment was repeated only by Babović and Mekić in 2011 [9] and a full analysis of its motion is given in the reference [11.

Another interesting experiment with the Foucault pendulum was recalled by Cox [12] in 1951 during the centennial anniversary of Foucault pendulum. An additional rotating frame was used which, together with the Foucault pendulum, rotated with the same angular velocity of the frame in rotation with the Earth, thus simulating an inertial frame. It was found that the trajectory described in such a rotating frame was a high eccentricity ellipse. In addition, as stated by the Chevilliet theorem, the ratio between the major and minor axes of the ellipse is the ratio between the periods of Earth's rotation and the pendulum swing.

The theoretical study of the pendulum motion is well known and is frequently done from Newton's second law in the frame rotating with the Earth. The exact solution of Newton's differential equations in the threedimensional Cartesian coordinates is relatively elaborate to obtain [11. However, as it is well known and shown in this paper, very good approximations can be made to simplify the mathematical calculations.

The purpose of this text is to present a review on the Foucault pendulum motion in the ideal case of small amplitudes of oscillation and without external interferences. The text shows the frame choices, Newton's 
equations in Cartesian coordinates in the rotating frame and the calculations that describe the Foucault pendulum motion, justifying all approximations used for mathematical calculations. The article also shows the pendulum trajectories in the two referential frames and contributes with a new method to determine the angular velocity, precession period of the pendulum and its displacement in each oscillation, based on the angular position of cusps of the Foucault pendulum trajectory. The pendulum motion is discussed for the case where the pendulum bob has an initial velocity. In addition, the Chevilliet theorem is demonstrated.

This text is useful for students to follow many details on the motion of the Foucault pendulum in inertial and rotational frames and help in the study of the particle motion in frames performing rotation and translation.

\section{Frames and the Equation of Motion}

In the analysis of a particle motion in rotating frames, most of the texts in classical mechanics consider that the inertial and non-inertial frames have coinciding origins [13. In this case the equation of motion of the mass particle, $m$, subject to external force, $\vec{F}$, in a rotating frame with constant angular velocity, $\vec{\omega}$, is written as:

$$
m \vec{a}^{\prime}=\vec{F}-m \vec{\omega} \times\left(\vec{\omega} \times \vec{r}^{\prime}\right)-2 m \vec{\omega} \times \vec{v}_{r}
$$

where $\vec{a}^{\prime}, \vec{r}^{\prime}$ and $\vec{v}_{r}$ are respectively the acceleration, the position and velocity of the particle in the rotating frame. The term $-m \vec{\omega} \times\left(\vec{\omega} \times \vec{r}^{\prime}\right)$ is the centrifugal force, and the term $-2 m \vec{\omega} \times \vec{r}_{r}$ is the Coriolis force.

For practical reasons, in the study of particle motion close to the Earth's surface, as shown in Fig. 1 the origin of the non-inertial frame $\left(O^{\prime}, x^{\prime}, y^{\prime}, z^{\prime}\right)$ is placed at the Earth's surface and the origin of the inertial frame $(O, x, y, z)$ in the center of the Earth.

Since non-coinciding origins are used for the inertial and rotating frames, it is necessary to adapt Eq. (1) to this new situation, in which the origin $O^{\prime}$ is displaced by the vector $\vec{R}$. Now, the reference $\left(O^{\prime}, x^{\prime}, y^{\prime}, z^{\prime}\right)$ translates due to the Earth's rotation and the centrifugal acceleration of $O^{\prime}, d^{2} \vec{R} / d t^{2}=-\vec{\Omega}_{T} \times\left(\vec{\Omega}_{T} \times \vec{R}\right)$, is omitted, being the vector $\vec{\Omega}_{T}$ is the Earth angular velocity. Therefore, Eq. (1) must take into account this acceleration, resulting in:

$$
\begin{aligned}
m \vec{a}^{\prime}= & \vec{F}-m\left[\vec{\omega} \times\left(\vec{\omega} \times \vec{r}^{\prime}\right)+\vec{\Omega}_{T} \times\left(\vec{\Omega}_{T} \times \vec{R}\right)\right] \\
& -2 m \vec{\omega} \times \vec{v}_{r}
\end{aligned}
$$

Vector $\vec{\omega}$ is the angular velocity of the reference $\left(O^{\prime}, x^{\prime}, y^{\prime}, z^{\prime}\right)$ in rotation, which depends on the latitude of $O^{\prime}$ on the Earth's surface, as will be shown later. It is worth mentioning that the angular velocity vector is invariant to a translation of the coordinate frame.

The modulus of $\vec{R}$, the Earth radius, is $\sim 6.4 \times 10^{6} \mathrm{~m}$, being much larger than the vector module $\vec{r}^{\prime}$ (of the order of meters). Therefore, the term $\vec{\omega} \times\left(\vec{\omega} \times \vec{r}^{\prime}\right)$ in Eq. (2) can be neglected for sure. With this approximation and separating the weight force, $\vec{P}=m \vec{g}$, from the external force, $\vec{F}$, in Eq. (2):

$$
m \vec{a}^{\prime}=\vec{F}+m\left[\vec{g}-\vec{\Omega}_{T} \times\left(\vec{\Omega}_{T} \times \vec{R}\right)\right]-2 m \vec{\omega} \times \vec{v}_{r}
$$

that is:

$$
m \vec{a}^{\prime}=\vec{F}+m \vec{g}_{e}-2 m \vec{\omega} \times \vec{v}_{r}
$$

where $\vec{g}_{e}=\vec{g}-\vec{\Omega}_{T} \times\left(\vec{\Omega}_{T} \times \vec{R}\right)$ is the effective acceleration vector of Earth's gravity, pointing in the direction of the plumb line, slightly dependent on the latitude of $O^{\prime}$ because the Earth is not perfectly spherical. Usually, the value of $\left|\vec{\Omega}_{T} \times\left(\vec{\Omega}_{T} \times \vec{R}\right)\right|$ is $\approx 3.4 \times 10^{-2} \mathrm{~m} / \mathrm{s}^{2}$, i.e., $\approx 0.3 \%$ of the value of $g$. This means that the $z^{\prime}$ axis, in the vertical direction at the Earth's surface, is redefined in the direction of the plumb line, which is practically the vertical direction.

Before performing the calculations, it is necessary to write the angular velocity, $\vec{\omega}$, of the frame $\left(O^{\prime}, x^{\prime}, y^{\prime}, z^{\prime}\right)$ in rotation with the Earth, in terms of latitude and the axes $\left(\hat{i}^{\prime}, \hat{j}^{\prime}, \hat{k}^{\prime}\right)$ of this coordinate frame. In Fig. 1 it is assumed that the reference is situated in the Northern Hemisphere, and since the axis of $x^{\prime}$ is tangent to the Earth's parallel (perpendicular to $\vec{\Omega}_{T}$ ), it follows that:

$$
\vec{\omega}=\Omega_{T}\left(-\cos \lambda \hat{j}^{\prime}+\sin \lambda \hat{k}^{\prime}\right)
$$

where $\lambda$ is the latitude of point $O^{\prime}$ on Earth.

The external force, $\vec{F}$, in Eq. (4) is the force, $\vec{T}$, exerted by the string on the pendulum bob. Using the geometry of Fig. 2 .

$$
\vec{T}=-T \sin \theta \cos \beta \hat{i}^{\prime}-T \sin \theta \operatorname{sen} \beta \hat{j}^{\prime}+T \cos \theta \hat{k}^{\prime}
$$

where the angles $\theta$ and $\beta$ are defined in the figure.

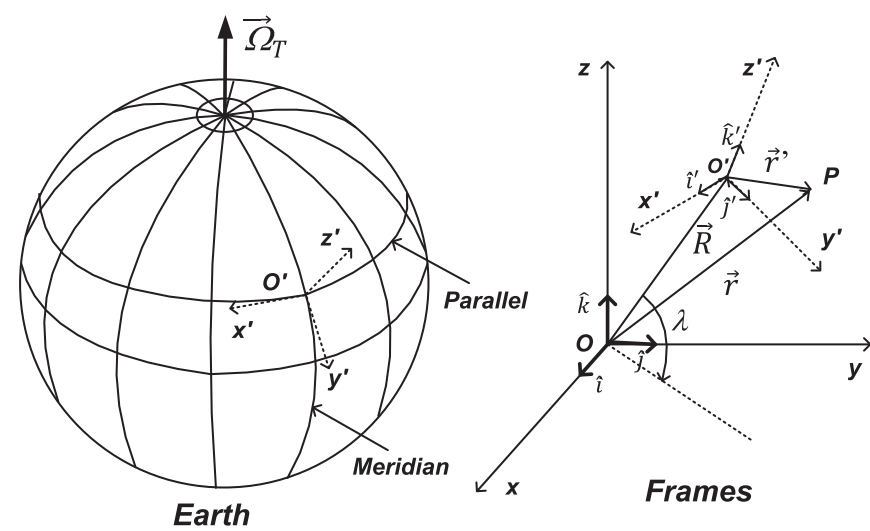

Figure 1: Frames to describe the motion of a particle $P$ in the Northern Hemisphere. The inertial frame $(O, x, y, z)$ is fixed at the center of the Earth and the rotating frame with the Earth is $\left(O^{\prime}, x^{\prime}, y^{\prime}, z^{\prime}\right)$. The plane $\left(x^{\prime}, y^{\prime}\right)$ coincides with the horizontal plane at the Earth's surface and $z^{\prime}$ is the vertical direction. The angle $\lambda$ is the latitude of $O^{\prime}$ on Earth. 


\section{Differential equations and their solutions in the Earth frame}

In order to write the differential equation for the pendulum bob (considered as a particle) in Cartesian coordinates, we start from Eq. (4) with reference to Fig. 2, which shows the pendulum arrangement, of length $L$, in $\left(O^{\prime}, x^{\prime}, y^{\prime}, z^{\prime}\right)$ in the rotation frame with the Earth. Using Eqs. (4) and (6), $\vec{g}_{e}=-g_{e} \hat{k}^{\prime}$ and the relations $\cos \beta=x^{\prime} / L \operatorname{sen} \theta$ e sen $\beta=y^{\prime} / L \operatorname{sen} \theta$, obtained from the geometry of Fig. 2 .

$m \vec{a}^{\prime}=-m g_{e} \hat{k}^{\prime}-T \frac{x^{\prime}}{L} \hat{i}^{\prime}-T \frac{y^{\prime}}{L} \hat{j}^{\prime}+T \cos \theta \hat{k}^{\prime}-2 m \vec{\omega} \times \vec{v}_{r}$

A second approximation is necessary to simplify this equation. For a very long pendulum that oscillates with small amplitude, one has $\cos \theta \cong 1$ and $T \cong$ $P \cong m g_{e}$. The validity of this approximation using numerical values for the Foucault pendulum is discussed in Section 4 Using these approximations and dividing Eq. (7) by the mass, the acceleration of the pendulum bob in the frame $\left(O^{\prime}, x^{\prime}, y^{\prime}, z^{\prime}\right)$ is:

$$
\vec{a}^{\prime}=-g_{e} \frac{x^{\prime}}{L} \hat{i}^{\prime}-g_{e} \frac{y^{\prime}}{L} \hat{j}^{\prime}-2 \vec{\omega} \times \vec{v}_{r}
$$

Using $\vec{v}_{r}=\dot{x}^{\prime} \hat{i}^{\prime}+\dot{y}^{\prime} \hat{j}^{\prime}+\dot{z}^{\prime} \hat{k}^{\prime}, \vec{a}^{\prime}=\ddot{x}^{\prime} \hat{i}^{\prime}+\ddot{y}^{\prime} \hat{j}^{\prime}+\ddot{z}^{\prime} \hat{k}^{\prime}, \vec{\omega}$ from Eq. (5) to perform the vector product of the Coriolis acceleration, and equating the components in the directions $\left(\hat{i}^{\prime}, \hat{j}^{\prime}, \hat{k}^{\prime}\right)$, gives:

$$
\begin{aligned}
& \ddot{x}^{\prime}=-\omega_{0}^{2} x^{\prime}+2 \Omega_{T}\left(\dot{z}^{\prime} \cos \lambda+\dot{y}^{\prime} \sin \lambda\right) \\
& \ddot{y}^{\prime}=-\omega_{0}^{2} y^{\prime}-2 \Omega_{T} \dot{x}^{\prime} \sin \lambda \\
& \ddot{z}^{\prime}=-2 \Omega_{T} \dot{x}^{\prime} \cos \lambda
\end{aligned}
$$

where $\omega_{0}=\sqrt{g_{e} / L}$ is the angular frequency of the pendulum oscillation. The dot on top of the variables

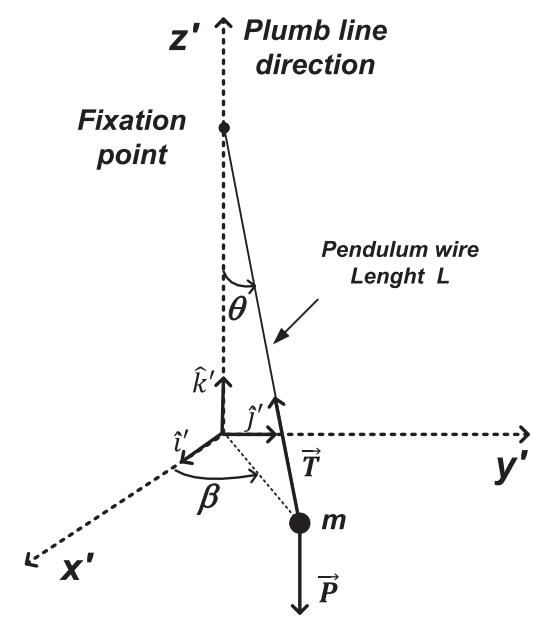

Figure 2: Diagram of the pendulum in the frame $\left(O^{\prime}, x^{\prime}, y^{\prime}, z^{\prime}\right)$ rotating with the Earth, with angular velocity $\vec{\omega}$. in Eq. (9) represents the derivative with respect to time (velocity), while two dots mean the second derivative (acceleration). Differential equations are typical of an oscillatory movement, but they are coupled because they contain velocities in the other directions of motion.

At this point the third approximation is used: motion occurs in the horizontal plane $\left(x^{\prime}, y^{\prime}\right)$ of the frame $\left(O^{\prime}, x^{\prime}, y^{\prime}, z^{\prime}\right)$, that is, $z^{\prime}=\dot{z}^{\prime}=\ddot{z}^{\prime}=0$. Considering a planar motion is also a good approximation, as discussed in Section 4 using numerical values. Thus, the equations for the pendulum bob are simplified to:

$$
\begin{aligned}
& \ddot{x}^{\prime}=-\omega_{0}^{2} x^{\prime}+2 \Omega \dot{y}^{\prime} \\
& \ddot{y}^{\prime}=-\omega_{0}^{2} x^{\prime}-2 \Omega \dot{x}^{\prime}
\end{aligned}
$$

where $\Omega=\Omega_{T}$ sen $\lambda$ is the pendulum angular velocity around the $z^{\prime}$ axis and $\lambda$ is the latitude of $\left(O^{\prime}, x^{\prime}, y^{\prime}, z^{\prime}\right)$ on the Earth's surface. To solve these coupled differential equations, the second equation is multiplied by the complex number $i$ and the resulting equation is added to the equation of $\ddot{x}^{\prime}$ of Eq. 10. Then, the substitution of $z^{\prime}=x^{\prime}+i y^{\prime}$, provides:

$$
\ddot{z}^{\prime}+i 2 \Omega \dot{z}^{\prime}+\omega_{0}^{2} z^{\prime}=0
$$

whose solution includes $e^{i p t}$. The general solution to this equation can be written as:

$$
z(t)=A e^{i \alpha t}+B e^{-i \beta t}
$$

where $A$ and $B$ are constants and $\alpha$ and $\beta$ are:

$$
\alpha=-\Omega+\sqrt{\Omega^{2}+\omega_{0}^{2}} \text { and } \beta=\Omega+\sqrt{\Omega^{2}+\omega_{0}^{2}}
$$

The constants $A$ and $B$ are determined from the initial conditions of the motion. Although the movement of Foucault pendulum is initiated from rest $\left(\dot{x}^{\prime}(0)=\right.$ $\left.\dot{y}^{\prime}(0)=0\right)$, the initial conditions are assumed to be somewhat more general: $y^{\prime}(0)=0, x^{\prime}(0)=x_{0}$ and $\dot{x}^{\prime}(0)=0, \dot{y}^{\prime}(0)=v_{0 y}$. That is, the pendulum bob is assumed to have an initial velocity in the direction perpendicular to the plane of oscillation. Starting the pendulum movement along the $x^{\prime}$ axis $\left(y^{\prime}(0)=0\right)$ does not introduce any particularity to the study, but helps to simplify the algebraic work to obtain $x^{\prime}(t)$ and $y^{\prime}(t)$.

After the algebraic work to determine the constants $A$ and $B$, the coordinates of the motion in the frame with the Earth are:

$$
\begin{aligned}
& x^{\prime}(t)=\frac{1}{\alpha+\beta}\left[\left(\beta x_{0}+v_{0 y}\right) \cos \alpha t+\left(\alpha x_{0}-v_{0 y}\right) \cos \beta t\right] \\
& y^{\prime}(t)=\frac{1}{\alpha+\beta}\left[\left(\beta x_{0}+v_{0 y}\right) \sin \alpha t-\left(\alpha x_{0}-v_{0 y}\right) \sin \beta t\right]
\end{aligned}
$$

\section{Motion of the Foucault Pendulum in the Earth Frame}

In order to generate the trajectory curves we used a pendulum with the same characteristics used by 
Foucault in 1851: oscillation amplitude, $x_{0}=3 \mathrm{~m}$, angular frequency $\omega_{0}=\sqrt{g_{e} / L}=0.38 \mathrm{~s}^{-1}(L=67$ $\mathrm{m}$ and $\left.g_{e}=9.8 \mathrm{~ms}^{-2}\right)$, Paris latitude of $48.86^{\circ}$ and Earth angular velocity $\Omega_{T}=7.27 \times 10^{-5} \mathrm{~s}^{-1}$. The simulations to obtain the trajectories were performed using the program MathCad ${ }^{\circledR}$.

Before showing the results, the validity of the approximations in Section 3 is discussed. The approximation $\cos \theta \cong 1$ to simplify Eq. (7) is excellent since for the maximum oscillation amplitude of $3 \mathrm{~m}$, one has $\cos \theta=0.999$. The approximation of considering a planar motion to simplify Eq. 10 is also very good. Foucault pendulum was $67 \mathrm{~m}$ long, oscillated with the maximum angle of 2.6 degrees and rose up to $\approx 6 \mathrm{~cm}$ with respect to the lowest point of the bob, that is, $\approx 0.1 \%$ of its length. To simplify some of the calculations in what follows, the quadratic term in $\Omega$ will be neglected since $\omega_{0}^{2} \gg \Omega^{2}$. This is also an excellent approximation because $\omega_{0}^{2}=$ $0.15 \mathrm{~s}^{-2}$ while $\Omega^{2} \leq 5.3 \times 10^{-9} \mathrm{~s}^{-2}$.

\subsection{Foucault pendulum trajectory}

Figure 3 shows the bob path of Foucault pendulum in the frame with the Earth, calculated from $x^{\prime}(t)$ and $y^{\prime}(t)$ from Eq. (14, with $v_{0 y}=0$. Note that the scale of $x^{\prime}$ axis in the figure is given in meters while the scale for $y^{\prime}$ is in centimeters. The time of simulation corresponds to approximately three periods of oscillation $(50 \mathrm{~s})$.

Figure 3 shows that the pendulum bob precess in the clockwise direction and at the reversal points (at the maximum amplitude of the oscillation) the trajectory has the cusp shape (points $A$ and $B$ in the figure). At these points the pendulum bob is at rest, which is why the trajectory has the shape of a star. The motion of Foucault pendulum depicted in Fig. 3 is characteristic of the initial condition of zero transverse velocity in the Earth frame. The movement begins in

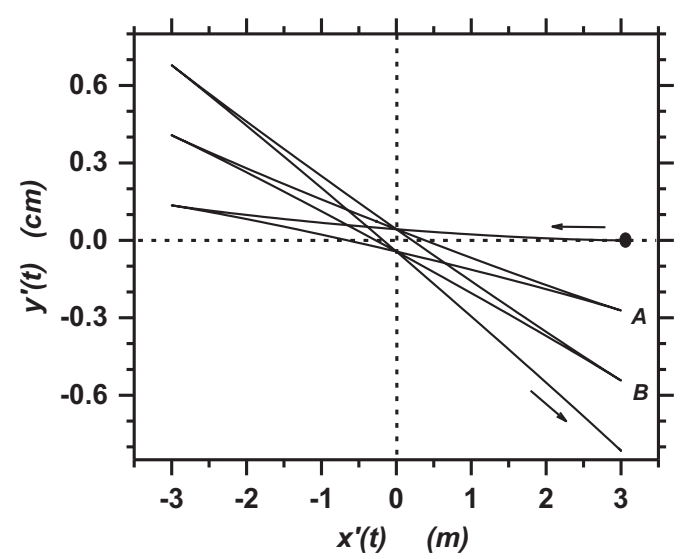

Figure 3: The bob trajectory of the Foucault pendulum in the Earth frame in Paris $\left(v_{0 y}=0\right)$. The total oscillation time is 50 $\mathrm{s}$ and the arrows indicate the direction of pendulum oscillation. The numerical values are those described in the text. $A$ and $B$ represent two consecutive cusps. the negative direction of $x^{\prime}$ axis but shifts slightly to the right due to the horizontal component of the Coriolis force $\left(-2 m \vec{\omega} \times \vec{v}_{r}\right)$. Upon reaching the reversal point of movement (at the cusp), where the velocity cancels out; the pendulum starts the movement back, diverting again to the right. From Fig. 3 one also notes that the pendulum bob does not pass through the origin of the coordinate frame, which is the center of the forces.

\subsection{Calculation of precession velocity and deviation at each oscillation}

The precession period of Foucault pendulum has been determined with geometric methods as in reference [14, 15] or in the book of Symon [13] that uses three coordinate frames or polar coordinates [16. However, to the best of my knowledge for Cartesian coordinates the determination of precession period cannot be performed from the coordinates $x^{\prime}(t)$ and $y^{\prime}(t)$. In this work, a procedure is presented based on the angular positions of the cusps in Fig. 3 Since the cusp positions belong to the circle of radius equal to the maximum amplitude of oscillation, the angular velocity of the pendulum precession is determined without difficulty from the angular cusps position.

The two consecutive cusps $A$ and $B$ (see Fig. 4) appear at each oscillation period, that is, they appear at the instants $t_{n}=\left(2 \pi / \omega_{0}\right) n$, where $n=0,1,2, \ldots$ The angle formed with the $x^{\prime}$ axis by the $n$-th cusp can be calculated through the coordinates $x^{\prime}(t)$ and $y^{\prime}(t)$. For $t_{n}=\left(2 \pi / \omega_{0}\right) n, v_{0 y}=0$ and $\omega_{0}^{2} \gg \Omega^{2}$, Eq. (14) gives:

$$
\begin{aligned}
& x_{n}^{\prime}\left(t_{n}\right)=x_{0} \cos \left(2 \pi \frac{\Omega}{\omega_{0}} n\right) \\
& y_{n}^{\prime}\left(t_{n}\right)=x_{0} \operatorname{sen}\left(2 \pi \frac{\Omega}{\omega_{0}} n\right)
\end{aligned}
$$

The angle $\theta$ with $x^{\prime}$ axis of the $n$-th cusp is:

$$
\tan \theta=\frac{y_{n}^{\prime}\left(t_{n}\right)}{x_{n}^{\prime}\left(t_{n}\right)}=-\tan \left(2 \pi \frac{\Omega}{\omega_{0}} n\right),
$$

that is,

$$
\theta=-2 \pi \frac{\Omega}{\omega_{0}} n
$$

and the angular variation between two consecutive cusps is:

$$
\Delta \theta=-2 \pi \frac{\Omega}{\omega_{0}}
$$

thus, the modulus of the angular velocity and the period of precession are:

$$
\begin{aligned}
& \omega_{P}=\frac{\Delta \theta}{T}=\frac{\Delta \theta}{2 \pi / \omega_{0}}=\Omega=\Omega_{T} \sin \lambda \text { and } \\
& T_{P}=\frac{2 \pi}{\Omega_{T} \sin \lambda}
\end{aligned}
$$


The deviation of Foucault pendulum at each oscillation is also easily calculated by:

$$
\Delta d=x_{0} \Delta \theta=2 \pi x_{0} \frac{\Omega}{\omega_{0}}=2 \pi x_{0} \frac{\Omega_{T} \operatorname{sen} \lambda}{\omega_{0}}
$$

At the Earth's poles (latitude $\lambda=\pi / 2$ ), the Foucault pendulum would return to its original position in $24 \mathrm{~h}$ $\left(T_{p}=2 \pi / \Omega_{T}\right)$ and at the Equator $(\lambda=0)$ the pendulum would not present precession motion $\left(T_{p} \rightarrow \infty\right)$. For the city of Paris, latitude of $+48.86^{\circ}$, the precession period of Foucault pendulum (clockwise) is $31 \mathrm{~h}$ and $53 \mathrm{~min}$ and for each oscillation period it deflects $2.7 \mathrm{~mm}$. These values agree with the results by Foucault in 1851 (period of $31 \mathrm{~h}$ and $47 \mathrm{~min}$ and deviation of $2.3 \mathrm{~mm}$ ).

Table 1 lists the precession periods (counterclockwise) that a pendulum, with the same characteristics used by Foucault, would have in some South American cities at different latitudes. In places near Equator, such as Manaus, the precession period is too long to be observed and measured. Further south in Buenos Aires, the precession period is about two days. The long periods in Table 1 indicate the difficulty to set Foucault pendulum in South America, because in the most cities the pendulum precesses too slowly to fascinate the public. Furthermore, energy dissipation and the pendulum suspension become important issues to build the pendulum.

Table 1: Precession periods of Foucault pendulum in South American cities at different latitudes.

\begin{tabular}{lcc}
\hline City & Latitude (degrees) & Period (hours) \\
\hline Manaus & -3.11 & 442 \\
Rio de Janeiro & -22.54 & 62.6 \\
São Paulo & -23.55 & 60 \\
Porto Alegre & -30.03 & 48 \\
Bueno Aires & -34.60 & 42 \\
\hline
\end{tabular}

\subsection{Calculation of bob velocity}

To complete the description of the Foucault pendulum motion it is also instructive to calculate the bob velocity. For this purpose the derivative related to time of $x^{\prime}(t)$ and $y^{\prime}(t)$ of Eq. (14) are taken with $v_{0 y}=0$. The components $\dot{x}^{\prime}(t)$ and $\dot{y}^{\prime}(t)$ of the velocity are:

$$
\begin{aligned}
& \dot{x}^{\prime}(t)=\frac{\alpha \beta x_{0}}{\alpha+\beta}(\sin \alpha t+\sin \beta t) \\
& \dot{y}^{\prime}(t)=\frac{\alpha \beta x_{0}}{\alpha+\beta}(\cos \alpha t-\cos \beta t)
\end{aligned}
$$

The slope of the trajectory of the pendulum bob is determined, giving:

$$
\frac{d y^{\prime}}{d x^{\prime}}=\frac{d y^{\prime} / d t}{d x^{\prime} / d t}=\frac{\dot{y}^{\prime}(t)}{\dot{x}(t)}=\frac{\cos \alpha t-\cos \beta t}{\sin \alpha t+\sin \beta t}
$$

Using with $\alpha$ and $\beta$ from Eq. (13):

$$
\frac{d y^{\prime}}{d x^{\prime}}=-\tan \Omega t
$$

from which it is concluded that initially the tangent to the trajectory has negative slope producing the deviation to the right of the movement, as shown in Fig. 3. Also, the deviation varies with the constant time-rate equal to $\Omega$ during the pendulum swinging, consistent with the Foucault pendulum precessing with this angular velocity (see Eq. (18)). After the pendulum precessed by $\pi / 2$, the slope becomes positive. After $\pi$, it is again negative and so on. But the deviation is always to the right of the movement.

The pendulum bob acceleration can be obtained taking the derivatives with respect to the time of $x^{\prime}(t)$ and $y(t)$ in Eq. 20 and using the approximations $\omega_{0}^{2} \gg \Omega^{2}$ and $\cos \Omega t=1$. At the beginning of the movement, when the pendulum practically oscillates along the $x^{\prime}$ axis, $\ddot{x}^{\prime}(t \rightarrow 0) \cong-x_{0} \omega_{0}^{2} \cos \omega_{0} t$ and $\ddot{y}^{\prime}(t \rightarrow 0) \cong 0$, which are the expected values for the acceleration of the oscillatory movement. When the pendulum precesses by $\pi / 2, \ddot{y}^{\prime}\left(t \rightarrow \frac{\pi}{2 \Omega}\right) \cong x_{0} \omega_{0}^{2} \cos \omega_{0} t$ and $\ddot{x}^{\prime}\left(t \rightarrow \frac{\pi}{2 \Omega}\right) \cong 0$, since it now oscillates along the $y^{\prime}$ axis.

\subsection{Pendulum with initial velocity}

When an initial velocity is impressed on the pendulum bob in the direction perpendicular to the plane of oscillation, the coordinates of motion are given in Eq. (14), with $\dot{y}(0)=v_{0 y}$. Herein, the case considered has the pendulum bob being launched with the initial velocity, $v_{0 y}=-x_{0} \Omega$. This value corresponds to the velocity of $O^{\prime}$ due to the Earth's rotation but in the opposite direction. Such initial condition implies that in the inertial frame the pendulum bob is abandoned from rest. Figure 4 shows the trajectory of the pendulum bob, which has a petals shape in the frame $\left(O^{\prime}, x^{\prime}, y^{\prime}, z^{\prime}\right)$. This differs from the case where the bob is abandoned from rest,

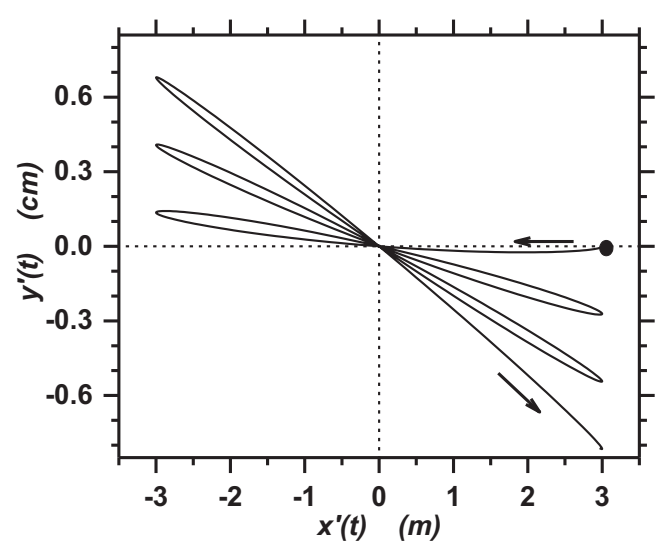

Figure 4: The bob trajectory of the Foucault pendulum in the Earth frame. The numerical data refer to the Foucault pendulum in Paris and the initial velocity was $v_{0 y}=-x_{0} \Omega=-0.16 \mathrm{~mm} / \mathrm{s}$. 
in which the trajectory has the shape of a star. As in the case without initial velocity, the pendulum precesses clockwise, but the pendulum bob always goes across the origin of the coordinate frame.

This change of shape is particularly intriguing because the initial velocity in the $y^{\prime}$ axis direction to obtain the trajectory of Fig. 4 $v_{0 y}=-x_{0} \Omega=-0.16 \mathrm{~mm} / \mathrm{s}$, is very small. Thus, any disturbance in abandoning the Foucault pendulum from rest will change the shape of the cusps. Instead of the shape in Fig. 3 the movement will have a trajectory in the form of petals if the bob is not abandoned from rest. Care must be taken to initiate the movement of the Foucault pendulum bob from rest, although for the Foucault pendulum deviations are too small to be observed in practice.

\section{Pendulum Motion in the Inertial Frame}

The motion of the Foucault pendulum is often explained with confusing arguments in textbooks [13, 17]. As Foucault's pendulum is released from rest in the rotating reference of Earth, it has a small initial velocity in the direction perpendicular to the plane of oscillation in the inertial frame. This makes the pendulum bob to perform an elliptical trajectory in the inertial frame, as proposed by the Chevilliet theorem. The bob path of the Foucault pendulum in the inertial frame is shown below and the Chevilliet's theorem will also be demonstrated.

The bob coordinates in the reference $(O, x, y, z)$ are obtained from the geometry in Fig. 5 where the frame $\left(O^{\prime}, x^{\prime}, y^{\prime}, z^{\prime}\right)$ rotates with the angular velocity $\Omega=$ $\Omega_{T} \operatorname{sen} \lambda$. From Fig. 5 we obtain:

$$
\begin{aligned}
& x(t)=x^{\prime}(t) \cos \Omega t-y^{\prime}(t) \sin \Omega t \\
& y(t)=x^{\prime}(t) \sin \Omega t+y^{\prime}(t) \cos \Omega t
\end{aligned}
$$

In Fig. 5 it was assumed that the reference origins are coincident, the axes directions were conveniently chosen and the inertial frame $(O, x, y, z)$ translates accompanying the movement of point $O^{\prime}$. In this configuration the centrifugal acceleration, $-\vec{\Omega}_{T} \times\left(\vec{\Omega}_{T} \times \vec{R}\right)$, of point

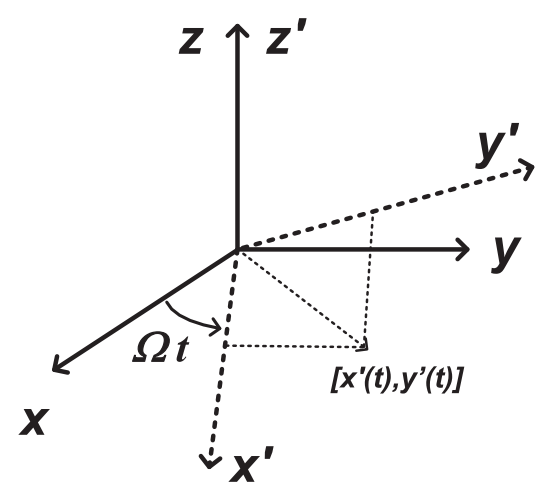

Figure 5: Diagram for coordinates transformation from the rotating frame to the inertial frame.
$O^{\prime}$ is neglected. However, it can be reasoned that it is included in the effective acceleration of gravity $\vec{g}_{e}$ as it was done to obtain Eq. (4). Thus, no information is lost in the calculations shown below.

Replacing $x^{\prime}(t)$ and $y^{\prime}(t)$ from Eq. (14) into Eq. (23), the coordinates $x(t)$ and $y(t)$ in the frame $(O, x, y, z)$ are:

$$
\begin{aligned}
& x(t)=x_{0} \cos \sqrt{\omega_{0}^{2}+\Omega^{2}} t \cong x_{0} \cos \omega_{0} t \\
& y(t)=\frac{\left(x_{0} \Omega+v_{0 y}\right)}{\omega_{0}} s \sqrt{\omega_{0}^{2}+\Omega^{2}} t \cong \frac{\left(x_{0} \Omega+v_{0 y}\right)}{\omega_{0}} \sin \omega_{0} t
\end{aligned}
$$

\subsection{Foucault Pendulum starting from rest}

The bob trajectory in the inertial frame in Fig. 5 for Foucault pendulum $\left(v_{0 y}=0\right)$ is a high eccentricity ellipse. This can be easily proven by eliminating the time from $x(t)$ and $y(t)$ of Eq. 24:

$$
\frac{x^{2}(t)}{x_{0}^{2}}+\frac{y^{2}(t)}{\left(\frac{x_{0} \Omega}{\omega_{0}}\right)^{2}}=1
$$

which represents the ellipse with eccentricity $e=$ $\sqrt{\frac{\omega_{0}^{2}-\Omega^{2}}{\omega_{0}^{2}}}$. For the case of the Earth frame, the approximation $\omega_{0}^{2} \gg \Omega^{2}$, gives an eccentricity of $\cong 1$, that is, in the inertial frame the trajectory of the pendulum movement is an ellipse with a high eccentricity as shown in Fig. 6. The figure also indicates that the bob starts the movement to the right side, which is the direction of Earth's rotation. The total displacement of the Foucault pendulum bob in the direction $y(t)$ is twice the value of the minor axis of the ellipse and equal to $\Delta y=2 x_{0} \Omega_{0}=$ $0.86 \mathrm{~mm}$. This agrees with the value of $1 \mathrm{~mm}$ measured in the experiment.

The Chevilliet theorem can be demonstrated by rewriting Eq. 25 in terms of the periods of motion:

$$
\frac{x^{2}(t)}{x_{0}^{2}}+\frac{y^{2}(t)}{\left(x_{0} \frac{T_{\Omega}}{T}\right)^{2}}=1
$$

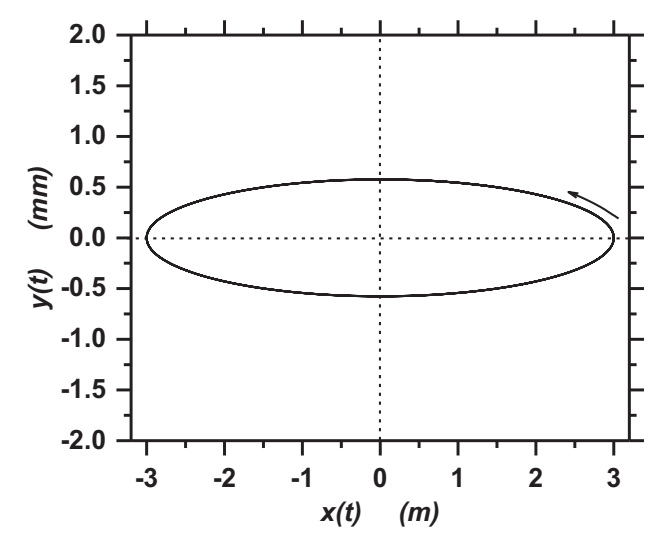

Figure 6: Elliptical trajectory of the Foucault pendulum in the inertial frame. The numerical values used refer to the Foucault pendulum in Paris. The arrow indicates the direction of bob movement. 
where $T_{\Omega}=2 \pi / \Omega$ and $T=2 \pi / \omega_{0}$. Using the definitions of the ellipse axes, the ratio between the major and minor axes is $x_{0} \frac{T_{\Omega}}{T} / x_{0}$. It is equal to the ratio $T_{\Omega} / T$ of the rotation period of the frame on the Earth with the period of Foucault pendulum, which amounts to a demonstration of the Chevilliet's theorem.

\subsection{Pendulum with initial velocity}

As already discussed, starting the Foucault pendulum bob with a small initial velocity, $v_{0 y}=-x_{0} \Omega=-0.16$ $\mathrm{mm} / \mathrm{s}$, in the frame $\left(O^{\prime}, x^{\prime}, y^{\prime}, z^{\prime}\right)$ in rotation with the Earth, is equivalent to abandoning the bob at the rest in the inertial frame $(O, x, y, z)$. Therefore, it will oscillate as a simple pendulum in the inertial frame, that is, always on the same plane, as shown in Fig. 7. This is easily proven from $x(t)$ and $y(t)$ in Eq. (24) by replacing $v_{0 y}=-x_{0} \Omega$ :

$$
x(t)=x_{0} \cos \omega_{0} t \quad \text { and } \quad y(t)=0
$$

Therefore, as $y(t)=0$, the pendulum swings in the inertial frame as a simple pendulum along the $x$ axis, as shown in Fig. 7

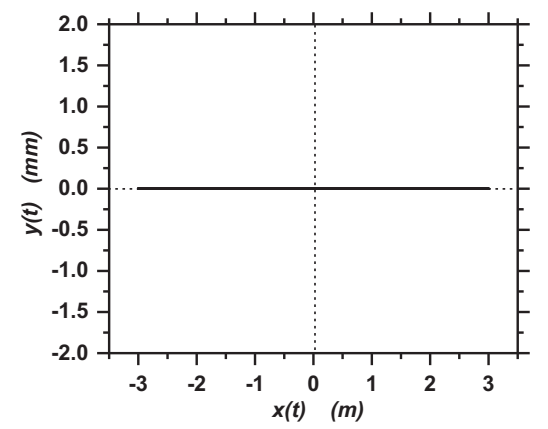

Figure 7: Linear trajectory for the Foucault pendulum in the inertial frame, launched with initial velocity $v_{0 y}=-x_{0} \Omega=$ $-0.16 \mathrm{~mm} / \mathrm{s}$. The numerical values used refer to the Foucault pendulum in Paris.

\section{Final Comments}

This paper brings together a full description of the Foucault pendulum motion in the inertial and rotating frames using Cartesian coordinates, for small amplitude oscillation and without any external interference. Approximations were used to determine pendulum motion: (a) the centrifugal acceleration $\left[-\vec{\omega} \times\left(\vec{\omega} \times \vec{r}^{\prime}\right)\right]$ was neglected compared to the acceleration $\left[-\vec{\Omega}_{T} \times\left(\vec{\Omega}_{T} \times \vec{R}\right)\right]$ due to Earth's rotation, and $(b)$ since the Foucault pendulum is very long, the differential equation is simplified and the bob motion is restricted to the horizontal plane on the Earth's surface. The new contribution here is the method to determine the angular velocity and the precession period of the Foucault pendulum from the angular position of cusps formed by the trajectory at the extreme points of the oscillation.

The solution to the Foucault pendulum motion can also be calculated using polar coordinates [16], which is a more appropriate system for writing the motion equations. More elaborated solutions are also known, for example, considering the three-dimensional motion in the Cartesian coordinates [11, 18. Also, other mechanic formalisms [18 20] are used to obtain the equations of motion. In summary, for more than a century and a half, the Foucault pendulum was the subject of very detailed studies.

To initiate the movement of the Foucault pendulum, care must be taken, since a small initial perturbation in the bob, in the direction perpendicular to the oscillation plane, modifies the pendulum trajectory to that shown in Fig. 4. although such small deviations are difficult to see in practice for the Foucault pendulum. However, other disturbances may be introduced into the pendulum motion, for example, by the electromagnetic mechanism used to compensate the energy loss or by the pendulum string support. They can cause an additional motion that changes the angular precession velocity, which is intensified when using a pendulum of very short length [21].

The expressions for the coordinates could differ from the ones given by Eq. (14) and produce some confusion to students when reading papers or books from different authors. For example, the approximation that $\omega_{0}^{2} \gg$ $\Omega^{2}$ could be used to simplify the notation. However, this approximation does not affect the results for the trajectory, because as discussed through this work it applies very well for the Foucault pendulum.

To finalize, it is also possible to consider the case of the Bravais pendulum mentioned in the introduction of the paper. The solution to Eq. (14) for the motion of the Foucault pendulum, with a suitable initial transversal velocity, also applies to the conical movement of the Bravais pendulum [11, as long as its length can be considered very long.

\section{Acknowledgments}

The author thanks Osvaldo N. Oliveira, Jr., for revising the final manuscript. Support from $\mathrm{CNPq}$ and INEO (Brazil) are also acknowledged.

\section{References}

[1] L. Foucault, Comptes Rendus Acad. Sci. 32, 135 (1851).

[2] J. Sommeria, Comptes Rendus Physique 18, 520 (2017).

[3] C.B. de Oliveira, Comptes Rendus Acad. Sci. 33, 582 (1851).

[4] R.P. Crease, World 15, 19 (2002).

[5] W. Tobin, The life and science of Léon Foucault - The Man who proved the world turned round (Cambridge University Press, Cambridge, 2002). 
[6] A.D. Aczel, Pendulum - Leon Foucault and the Triumph of Science (Atria Books, New York, 2003).

[7] M.A. Bravais, Comptes Rendus Acad. Sci. 33, 195 (1851).

[8] M.A. Bravais, Journal de Mathématiques Pures et Appliquées 1re série 19, 1 (1854)

[9] V.M. Babović and S. Mekić, Eur. J. Phys. 32, 1077 (2011).

[10] G. Barenboim and J.A. Oteo, Eur. J. Phys. 34, 1049 (2013).

[11] J.A. Giacometti, Am. J. Phys. 85, 292 (2020).

[12] J.F. Cox, L'Astronomie 65, 265 (1951).

[13] K.R. Symon, Mechanics (Addison-Wesley, Reading, 1963).

[14] J.B. Hart, R.E. Miller and R.L. Mills, Am. J. Phys. 55, 67 (1987).

[15] T.F. Jordan and J. Maps, Am. J. Phys. 78, 1188 (2010).

[16] W.J. Noble, Am. J. Phys. 20, 334 (1952).

[17] W.B. Somerville, Quarterly Journal of the Royal Astronomical Society 13, 40 (1972).

[18] F. Zhuravlev and A.G. Petrov, Doklady Physics 59, 35 (2014).

[19] R. Verreault, Eur. Phys. A. Appl. Phys. 79, 31001 (2017).

[20] D.M. Klimov, Mechanic of Solids 50, 371 (2015).

[21] H.R. Crane, Am. J. Phys. 49, 1004 (1981). 